\title{
Maternal negative affect during infancy is linked to disrupted patterns of diurnal cortisol \& alpha asymmetry across contexts during childhood
}

\section{Authors: Rebecca J. Brooker, Richard J. Davidson, and $\mathrm{H}$. Hill Goldsmith}

This is a postprint of an article that originally appeared in Journal of Experimental Child Psychology on October 2015.

Brooker, Rebecca J., Richard J. Davidson, and H. Hill Goldsmith. "Maternal negative affect during infancy is linked to disrupted patterns of diurnal cortisol \& alpha asymmetry across contexts during childhood." Journal of Experimental Child Psychology (October 2015). DOI: $10.1016 /$ j.jecp.2015.08.011 


\title{
Maternal negative affect during infancy is linked to disrupted patterns of diurnal cortisol and alpha asymmetry across contexts during childhood
}

\author{
Rebecca J. Brooker ${ }^{\mathrm{a}, *}$, Richard J. Davidson ${ }^{\mathrm{b}}$, H. Hill Goldsmith ${ }^{\mathrm{b}}$ \\ a Department of Psychology, Montana State University, Bozeman, MT 59718, USA \\ ${ }^{\mathrm{b}}$ Department of Psychology, University of Wisconsin-Madison, Madison, WI 53706, USA \\ * Corresponding author. E-mail address: rebecca.brooker@montana.edu (R.J. Brooker).
}

\begin{abstract}
Maternal negative affect in the early environment is believed to sensitize longterm coping capacities in children. Yet, little work has identified physiological systems associated with coping responses, which may serve as mechanisms for links between early maternal negativity and child outcomes. Using a longitudinal twin sample $(\mathrm{N}=89)$, we found that high levels of maternal negative affect during infancy were associated with dysregulation of diurnal cortisol and electroencephalograph (EEG) asymmetry, two physio- logical systems that may support active approach-oriented coping when children are 7 years old. Flattened slopes of diurnal cortisol were also associated with greater numbers of concurrent overanx- ious behaviors in children. A mediation analysis supported the role of dysregulated diurnal cortisol as a mediator of the link between maternal negative affect in the early environment and childhood risk for anxiety problems.
\end{abstract}

\section{Introduction}

High trait-level negative affect in mothers predicts negative psychological outcomes in children (Eisenberg et al., 2001; Weinberg \& Tronick, 1998), including increased risk for anxiety problems (Degnan, Almas, \& Fox, 2010). Yet, the mechanisms through which maternal negativity affects 
children's risk for anxiety problems are unclear. One possibility is that negativity in the early environment disrupts the biological systems that support the development of skilled coping or the execution of responses to perceived threat (Lazarus, 1966). The early sensitization hypothesis posits that socioemotional experiences in the early rearing environment program biological templates for coping responses that become prioritized over time (Gunnar \& Quevedo, 2007; Shirtcliff \& Ruttle, 2010). Under conditions of normative development, early experience programs responses that result in effective active coping behaviors (Sapolsky, 1998; Thompson, 1994). However, responses differ when programmed in non-normative environments. For example, high levels of maternal negativity in the early environment are associated with disrupted neuroendocrine function in preschoolers (Essex, Klein, Cho, \& Kraemer, 2003), which presumably handicaps active coping efforts (Gunnar, 1992) and increases early anxiety risk (Kagan, 1994). Here, we examined two physiological systems associated with active coping that may be disrupted by early experience and increase risk for anxiety problems during childhood.

\section{Maternal negativity and psychophysiological indices of active coping}

As described previously, the theory of early sensitization suggests that coping tendencies, programmed during infancy, are evident across development. Yet, little empirical work has tested longitudinal relations between infant experience and putative physiological underpinnings of coping in children. Extant research has largely focused on associations between maternal negative affect and hypothalamic-pituitary-adrenal (HPA) axis function. Activity of the HPA axis, measured via its end product cortisol, is heightened in response to environmental challenge (Miller \& O'Callaghan, 2002). Thus, cortisol may index, at the neuroendocrine level, active coping in the face of external stressors (Gunnar, 1992). In particular, well-regulated HPA axis function may support active, or problemfocused, coping. Active coping is characterized by individuals' adoption of an approach orientation toward problem solving and employment of direct actions to resolve challenge (Carver, Scheier, \& Weintraub, 1989). Such behaviors are more functional than avoidant strategies, in which efforts to deal with stressors are reduced, and are associated with improved mental health.

Regulation of the HPA system can be easily assessed. Cortisol follows a diurnal rhythm, discernible as early as 6 weeks of age, in which levels peak just after waking and reach a nadir near bedtime (Larson, White, Cochran, Donzella, \& Gunnar, 1998). Disruptions to the natural diurnal rhythm of cortisol, visible as a flattened slope of change in cortisol levels across the day, are associated with exposure to early environmental stressors (Gunnar \& Vazquez, 2001; Heim, Ehlert, \& Hellhammer, 2000). For example, children who experienced early maltreatment showed altered patterns of diurnal cortisol in the form of blunted morning peaks along with smaller decreases (i.e., flatter slopes) in cortisol levels across the day (Carlson \& Earls, 1997; Gunnar \& Vazquez, 2001). Disrupted HPA function may signal a diminished capacity to engage active coping strategies (Gunnar, 1992). Thus, individual capacities for coping may be disrupted at a systemic level as a result of early experience.

Children in non-extreme but highly negative early environments may also show disrupted diurnal cortisol rhythms, although this possibility is understudied. However, given that heightened negative affect in mothers is associated with poorer parenting quality (Lovejoy, Graczyk, O'Hare, \& Neuman, 2000), maternal negativity, even at non-extreme levels, may be a salient environmental factor for children during infancy. If this is the case, trait-level negativity in mothers should predict dysregulated diurnal cortisol rhythms in typically developing children. Thus, one of our goals was to examine the degree to which maternal negative affect during infancy predicted disrupted diurnal cortisol during childhood.

As noted previously, an important aspect of active coping is an approach orientation to dealing with environmental stress or challenge (Carver et al., 1989) given that avoidant coping strategies are linked to greater numbers of anxiety symptoms (Blalock \& Joiner, 2000). Approach and avoidance orientations are visible as differences in alpha power between the left and right hemispheres of the prefrontal cortex or frontal asymmetry. Greater baseline activity in the right, relative to the left, frontal hemisphere (i.e., right frontal asymmetry) denotes a disposition for heightened avoidance behaviors (Davidson \& Irwin, 1999). Similarly, greater activity in the left, relative to right, frontal hemisphere (i.e., left frontal asymmetry) denotes propensities for approach. 
Logically, then, one might expect to see the greatest levels of left frontal asymmetry in contexts that necessitate more coping. However, studies frequently find that highly challenging contexts elicit greater right, rather than left, frontal asymmetry (e.g., Buss et al., 2003). One possible explanation for these seemingly counterintuitive findings comes from the neuroendocrine literature. Neuroendocrine researchers have suggested that coping efforts are thwarted by greater uncontrollability and social threat in the environment, a possibly adaptive function of frontally mediated feedback mechanisms that prevent exhaustion of physiological resources in contexts that appear unlikely to be resolved through coping efforts (Laborit, 1976; Munck, Guyre, \& Holbrook, 1984; Tops et al., 2004). Thus, a more nuanced hypothesis regarding expectations about frontal asymmetry is necessary. That is, greater left frontal asymmetry should be anticipated in mild to moderately stressful contexts, where coping efforts are not reduced, whereas right frontal asymmetry should be expected in moderately to highly stressful environments.

The evidence just reviewed suggests that measures of frontal asymmetry in emotion-eliciting contexts offer unique information about the balance of context-based challenges and individuals' coping efforts (Coan, Allen, \& McKnight, 2006; Davidson, 2002). For example, contexts characterized by threat and uncertainty, such as interactions with unfamiliar people, are challenging for young children and are thus useful for examining individual differences in systems that support approach/ avoidance coping orientations. During infancy, stranger interactions elicit withdrawal and right frontal asymmetry (Buss et al., 2003), perhaps due to the high levels of stress elicited by such a novel and putatively threatening context. By preschool, regular interactions with strangers appear to be less threatening for children, who are increasingly able to appropriately engage in social contexts. Accordingly, many older children show greater left frontal asymmetry during social interactions (Fox et al., 1995). Thus, one might expect that during childhood, interactions with individuals who are more novel and hold greater levels of uncertainty present a greater social challenge. Such interactions would elicit greater left frontal asymmetry as children engage physiological systems that support active coping efforts.

Early sensitization theory would suggest that patterns of left frontal asymmetry resulting from changing contextual demands would be altered in children who experienced high levels of maternal negativity during infancy. Although this possibility has not been examined in a way that pairs infant experience with childhood outcomes, evidence suggests that infants of highly negative mothers show greater right frontal asymmetry during stranger interactions (Dawson et al., 1999) and less left frontal asymmetry during mother-child interactions (Dawson et al., 1999) relative to infants of less negative mothers. Similarly, preschoolers with mothers high in negativity show more right frontal asymmetry than preschoolers with less negative mothers (Jones, Field, \& Davalos, 2000). Thus, frontal asymmetry, an index of approach or withdrawal tendencies that are important for active coping, may be affected by the early rearing environment. Therefore, our second goal was to examine the degree to which early maternal negative affect during infancy predicted disrupted patterns of frontal asymmetry during periods of uncertainty in typically developing children.

\section{Maternal negativity, physiological underpinnings of coping, and risk for anxiety problems}

A central tenet of early sensitization theory is that disruptions in the physiological systems that support active coping are tied to individual differences in psychological outcomes. Indeed, disrupted diurnal cortisol rhythms in children (Greaves-Lord et al., 2007) and adolescents (Van den Bergh, Van Calster, Pinna Puissant, \& Van Huffel, 2008) are associated with more anxiety symptoms. However, such results are not entirely consistent in the literature. In a sample of nearly 300 middle-schoolaged children, flattened slopes of diurnal cortisol were linked to greater severity of pooled internalizing and externalizing symptoms but not to directionality of symptoms (i.e., preponderance of internalizing or externalizing symptoms independent of severity; Shirtcliff \& Essex, 2008). Thus, Shirtcliff and Essex (2008) suggested that flattened diurnal slopes are nonspecific to children's levels of anxiety. Given mixed evidence for associations between slopes of change in diurnal cortisol and anxiety symptoms during middle childhood, our third goal was to directly test the link between diurnal cortisol rhythms and anxious behaviors during middle childhood.

Given its link to avoidant coping behaviors, it is unsurprising that right frontal asymmetry has also been associated with early risk for anxiety problems. Propensities for withdrawal or avoidance are 
part of a behavioral profile that predicts risk for developing anxiety problems in young children (Biederman et al., 2001; Chronis-Tuscano et al., 2009). For children between 4.5 and 8 years of age, greater right frontal asymmetry during a baseline was associated with more internalizing symptoms, particularly for girls (Baving, Laucht, \& Schmidt, 1999). Thus, similar to diurnal cortisol, non-normative fluctuations in frontal asymmetry may index aspects of active (or avoidant) coping that are relevant for children's mental health outcomes. Therefore, a final aim of our study was to examine the link between fluctuations in frontal asymmetry across contexts and anxious behaviors during childhood.

\section{The current study}

Our goal was to test the degree to which maternal negative affect during infancy predicted disruptions in two physiological systems that support active coping during childhood. We tested our hypotheses across two systems in typically developing children. First, we examined whether an environment characterized by greater maternal negative affect during infancy was associated with slopes of diurnal cortisol during childhood. We hypothesized that greater maternal negative affect during infancy would be associated with dysregulated diurnal cortisol during childhood, visible as flatter slopes of change from morning to evening. Second, we tested whether greater maternal negative affect during infancy was associated with dysregulated patterns of frontal asymmetry in challenging contexts. Again, we hypothesized that greater maternal negative affect during infancy would be associated with more dysregulation in the form of flattened slopes of asymmetry across contexts that vary in approach incentive, indicating a lack of modulation of coping efforts to changes in the environment. Finally, we tested whether slopes indicating dysregulation of diurnal cortisol and frontal asymmetry were associated with individual differences in children's anxious behaviors. We hypothesized that, for both diurnal cortisol and frontal asymmetry, flatter slopes (indicating greater dysregulation) would be associated with greater numbers of anxious behaviors in children.

\section{Method}

\section{Participants}

Participants were drawn from a larger longitudinal study of emotional development in typically developing twins (Schmidt et al., 2013). Infant twin pairs were recruited from the greater Madison, Wisconsin, area in the midwestern United States through a variety of methods, including state birth records, mothers of twins clubs, television publicity, birth announcements in newspapers, doctors' offices, the Internet, and referrals from other participants. As a part of a larger study, 989 families were contacted for recruitment, which was ongoing throughout the study. A subset of families was invited to participate in a separate assessment aimed at examining affect, psychophysiology, and behavior when children were 7 years old. The current sample included participants from the infant study who provided usable electroencephalograph (EEG) or cortisol data at age 7 years $\left(N=89,53.9 \%\right.$ male). ${ }^{1}$

Participating families who provided demographic information $(n=82)$ were largely composed of Caucasian mothers (94.9\%) and fathers (94.7\%). Parent education ranged from 12 to 20+ years, although the most frequent reports were consistent with the completion of a college degree (16 years of education for $35.6 \%$ of mothers and fathers). Gross family income ranged from an interval of $\$ 20,001$ to $\$ 30,000$ to an interval of $\$ 100,001$ to $\$ 150,000$. The majority of families were middle class according to the Hollingshead index $(M=49.10, S D=9.35)$.

\section{Procedure}

When infants were 9 months old $(M=9.04, S D=0.91)$, caregivers were mailed a packet of questionnaires to complete and return to the laboratory via mail. When children reached 7 years of age

\footnotetext{
1 The total number of children who were enrolled in both assessments was $N=90$. One child provided no usable psychophysiological data and, thus, is not included in the current sample.
} 
( $M=7.45$ years, $S D=1.05$ ), they visited the laboratory, where EEG data were collected during a prebaseline episode (three emotion-eliciting episodes-conversation with experimenter, conversation with stranger, and stranger reading a script-involving a range of interactions with novel people) and a postbaseline episode. Consistent with the procedures of temperament studies designed to assess individual differences, episodes were presented in the same order for all children. Following the laboratory visit, parents collected saliva samples from children at home and returned samples to the laboratory.

\section{Measures}

\section{Maternal negative affect}

As a part of the 9-month mailing, mothers completed the Positive and Negative Affect Scale (PANAS), which is frequently used to assess trait levels of negative affect in adults (Watson, Clark, \& Tellegen, 1988). Unlike laboratory or observational measures that tend to capture emotional states or moods, the PANAS offers a trait-level quantification of maternal negativity during infancy. This conceptualization of maternal negativity is consistent with a notion of early sensitization. Mothers rated, using a 5-point interval scale, the degree to which they had experienced different emotions over the course of the previous few weeks $(1=$ very slightly or not at all, $5=$ extremely $)$. The 10 items $(\alpha=.87)$ asked about experiences of negative emotions, including "distressed, upset, irritable, and nervous." Maternal negative affect was scored as the sum of negative emotion item responses.

\section{Children's anxious behaviors}

At age 7 years, mothers and fathers rated their children's anxious behaviors on the overanxious scale of the Health and Behavior Questionnaire (HBQ; Essex et al., 2002). Each parent provided separate ratings of the degree to which a number of behaviors were true of each of their children $(0=$ never or not true, 2 = often or very true). Scores of overanxious behaviors were derived as the mean rating across 10 items that described behaviors consistent with a diagnosis of generalized anxiety (e.g., worries about future/past behavior, has trouble sleeping). Reliability was acceptable for both mothers $(\alpha=.74)$ and fathers $(\alpha=.68)$. Mother and father ratings were correlated $(r=.51, p<.01)$ and so were combined to improve reliability and reduce rater bias. Although the HBQ is designed to tap behaviors consistent with the diagnosis of anxiety disorders, it is not a diagnostic instrument; thus, high scores do not necessarily reflect clinical levels of anxiety.

\section{Diurnal cortisol}

Given evidence that noncompliance with instructions for sample collection can affect data validity, parents received detailed instructions describing how to collect three saliva samples per day from their children for 3 days at the following target times: within 30 min after waking, at $4 \mathrm{pm}$, and at children's bedtime before brushing teeth. Prior to leaving the laboratory, an experimenter instructed parents on saliva collection procedures, temporary sample storage (store in freezer), and return of samples to the laboratory (in a cooler bag). Written instructions that reiterated these points were sent home with families and included target times for each sample. The conical tube for each sample storage was labeled and color-coded to help parents distinguish the nine samples. As reported previously for this sample, the majority of morning samples were collected within a half hour of waking ( $M=0.53, S D=0.43$; Locke, Davidson, Kalin, \& Goldsmith, 2009), consistent with instructions. Roughly three quarters (76\%) of children provided the maximum nine usable samples.

The processing of saliva samples occurred in two stages. First, samples were thawed and centrifuged at $5000 \mathrm{rpm}$ for $10 \mathrm{~min}$ to extract the saliva from the cotton roll and then were transferred to a 2 -ml tube for storage $\left(-80^{\circ} \mathrm{C}\right)$. Then, the samples were divided into batches to be assayed. To minimize variability, all samples from each child were assayed within the same batch and were assayed with reagents from the same lot. Samples were assayed for cortisol with a high-sensitivity enzyme immunoassay kit (Salimetrics, State College, PA, USA) specifically designed for use with saliva. The sample test volume was $25 \mu \mathrm{l}$. The assay has a detection limit of $0.007 \mu \mathrm{g} / \mathrm{dl}$ and a range of sensitivity from 0.007 to $1.8 \mu \mathrm{g} / \mathrm{dl}$. Two internal controls were included in each assay. The average inter-assay coefficient of variation (CV) was 7.4\%, and the average intra-assay CV was $3.8 \%$. All samples were tested in duplicate within the same assay. The average of the duplicate tests was used in the 
analyses. Results were considered acceptable if the $\mathrm{CV}$ of the duplicate samples was less then or equal to $20 \%$. Repeat assays were performed on all samples not meeting this requirement.

Cortisol values were highly skewed, so outliers greater than 3 standard deviations from the mean were Windsorized. Cortisol values were regressed on sample time; residuals were saved and used for analysis. $^{2}$ All values were then log-transformed. Data were also screened for associations between cortisol values and sleep behavior, health status, and eating behavior. None of these variables was related to extreme cortisol values. Separate composites were created for morning (Days 1-3), afternoon (Days 1-3), and evening (Days 1-3) samples. The resulting three mean composites were used to calculate typical diurnal slopes.

\section{Frontal asymmetry}

EEG was recorded from 29 sites (13 homologous pairs and 3 midline sites) based on the 10 - to 20-electrode system during a baseline and three stranger interaction episodes. All impedances were reduced to less than $5 \mathrm{k} \Omega$ prior to recording. All channels were referenced online to physically linked ears and re-referenced offline to the average reference. Data were sampled at a rate of $200 \mathrm{~Hz}$ and amplified with a gain of 20,000. A $60-\mathrm{Hz}$ notch filter was applied during data collection to reduce electrical noise.

Data cleaning and segmentation procedures were conducted offline. Muscle artifact was removed using a low-pass filter with a high cutoff of $100 \mathrm{~Hz}$. Slow wave artifacts were removed using a highpass filter with a low cutoff of $0.1 \mathrm{~Hz}$. EEG data were first visually inspected for eye movement and blink artifacts. Additional artifacts were flagged using an automated procedure in MATLAB Data were then divided into 1 -min overlapping chunks (overlap $=0.5 \mathrm{~s}$ ). All 1-min chunks of artifact-free data were analyzed using a fast Fourier transform with a Hanning window. Alpha power values $(8-10 \mathrm{~Hz})$ were derived for each laboratory episode. The $8 \mathrm{~Hz}$ to $10 \mathrm{~Hz}$ frequency band was selected based on previous research with children in this age range (Baving et al., 1999; Hale et al., 2010; McManis, Kagan, Snidman, \& Woodward, 2002). Alpha power was highly correlated across frontal electrodes, and so mean composites were formed for left (F3, F7, Fc3, Fc7, FpF1) and right (F4, F8, Fc4, Fc8, FpF2) frontal alpha power. Frontal asymmetry scores were formed by subtracting the log transformed alpha power in the left composite from the log transformed alpha power in the right composite $\left(\mathrm{LOG} \alpha_{\text {right }}-\mathrm{LOG} \alpha_{\text {left }}\right)$. Calculated in this way, positive asymmetry scores reflect greater relative left frontal activation, whereas negative scores reflect greater relative right frontal activation. Scores of frontal asymmetry were calculated separately for each baseline and behavioral episode.

Baseline. The baseline episodes comprised eight 1-min resting periods, four with eyes open and four with eyes closed, in one of two counterbalanced orders. Children were instructed simply to sit quietly during each 1-min period. Seconds of usable EEG data from the baseline episode ranged from 51.60 to 60.31 per period $(M=58.99, S D=1.57)$.

Conversation with experimenter. In the conversation with experimenter episode, the primary experimenter entered the experimental room and spoke briefly from a script about the child's experiences in the laboratory that day, including discussions of toys that the child had seen, played with, and discussed previously with the experimenter. Seconds of usable EEG data from the conversation with experimenter episode ranged from 25.42 to $231.78(M=84.88, S D=33.40)$.

Conversation with stranger. An unfamiliar adult entered the laboratory room where EEG data were being collected and initiated a structured conversation with the child by asking him or her, in a neutral tone, "What do you like to do when you're not in school?" If the child did not respond to the stranger within $90 \mathrm{~s}$, the child was prompted with additional questions (e.g., "Could you tell me about what you like to do?"). The child was allowed to speak in response to the stranger until finished. When

\footnotetext{
${ }^{2}$ Consistent with the suggestions of previous research (Essex, Klein, Cho, \& Kalin, 2002), effects of mediation on cortisol levels were also examined; three children were excluded from the original data set, which included all children with data at age 7 years (i.e., not requiring children to have infant and childhood data) who were taking stimulant laxatives or insulin. Those children are also not present in the current sample.
} 
the child had finished speaking, or if the child had received three prompts without speaking, the stranger ended the episode. Seconds of usable EEG data from the stranger conversation episode ranged from 39.41 to $432.98(M=114.78, S D=66.91)$.

Stranger reading a script. In the stranger reading a script episode, the adult stranger from the conversation with a stranger episode followed his or her conversation with the child by reading a 1-min script to the child that explained basic aspects of the psychophysiology data collection procedures that had occurred during the child's visit to the laboratory. The stranger recited the script in a neutral tone. When the entire script had been read, the stranger thanked the child for listening and exited the experimental room. No response was requested or required from the child. Seconds of usable EEG data from the stranger reading a script episode ranged from 33.10 to $105.19(M=72.23, S D=11.23)$.

\section{Handedness}

Given evidence that patterns of hemispheric activation for cognitive and affective functions may differ in left- and right-handed individuals (Bryden, 1982), we restricted analyses using EEG data to the 74 children (83.1\% of the sample) who were right-handed, based on caregiver report. Analyses using cortisol data included the full sample of available participants $(N=89)$.

\section{Missing data}

Individuals meeting the aforementioned criteria (available 9-month and 7-year data) were included in the study $(N=89)$. EEG measures were missing for 6 participants $(6.7 \%)$, and cortisol data were missing for 11 participants (12.4\%). Roughly half of mothers $(47.2 \%)$ whose children participated in the 7-year physiology assessment had provided PANAS data when children were 9 months old.

Individuals with and without cortisol data did not differ on any of the variables $(|t| s<0.60$, $p s>.05)$. Similarly, children with and without EEG data did not differ on any of the variables ( $|t|$ $\mathrm{s}<1.90, p s>.05)$. Consistent with this, an analysis of patterns of missingness suggested that data were missing completely at random, ${ }^{3}$ Little's missing completely at random (MCAR): $\chi^{2}(182)=207.00$, $p>.05$. Thus, we imputed missing data $^{4}$ using a maximum likelihood expectation-maximization algorithm in SPSS 20.0 ( $n$ imputations $=100$ ). Reported parameter estimates are pooled estimates across all imputations. Asymmetry scores were not imputed for left-handed children.

\section{Plan for analysis}

All analyses were conducted using SAS Version 9.2 (SAS Institute, Cary, NC, USA). Following the examination of descriptive statistics, our hypotheses that maternal negative affect would disrupt regulatory systems were tested. Given the non-independent nature of twin data, a mixed analysis of variance (ANOVA) model was used to assess the interaction between psychophysiological regulation at age 7 years and maternal negative affect during infancy. Regulation (or dysregulation) was defined as either changes in diurnal cortisol across the day (Analysis 1 ) or alpha asymmetry across contexts (Analysis 2). Repeated assessments (alpha asymmetry or cortisol) were specified as within-person, time-varying predictors. Maternal negative affect (mean-centered) was included as a betweenparticipants, time-invariant predictor. Twins were identified as nested within families. Model fit statistics (Akaike information criterion [AIC] and Bayesian information criterion [BIC]) were used to determine the appropriateness of random effects.

\footnotetext{
${ }^{3}$ An analysis of additional demographic variables revealed that mothers with PANAS data tended to be higher in socioeconomic status $(M=52.04$ on the Hollingshead index) than mothers without PANAS data. Controlling for Hollingshead scores does not change the pattern of results reported here.

${ }^{4}$ The pattern of results is identical when analyses are run using a complete case analyses strategy. However, the risk for biased results is much greater if data are not imputed (Enders, 2010; Graham, 2009). Graham and Schafer (1999) showed that in even small samples $(N=50)$ with large multiple regression models (18 predictors) and large amounts of missing data (50\%), multiple imputation procedures produce results that are "as good as the same analyses performed on complete data" (Graham, 2009, p. 560). Thus, the multiple imputation procedure enhances our confidence in the current findings, particularly relative to a complete case analysis strategy.
} 
Following this, we tested our hypothesis that dysregulation of diurnal cortisol and alpha asymmetry were associated with children's risk for anxiety problems. To do this, individual slopes of change in psychophysiological measures (diurnal cortisol and alpha asymmetry) were extracted from individual-level regression analyses and tested as mediators of the link between maternal negativity at 9 months of age and overanxious behaviors at 7 years of age.

\section{Results}

\section{Descriptive statistics and sex differences among primary variables}

Descriptive statistics for variables are shown in Table 1. Consistent with previous literature, cortisol levels were greater during the morning than during the afternoon $(t=20.58, p<.01, d=2.84)$ or eve$\operatorname{ning}(t=13.11, p<.01, d=3.80)$. Consistent with a trend of steady declines in cortisol throughout the day, afternoon cortisol levels were greater than evening cortisol levels $(t=22.57, p<.01, d=1.57$ ).

Given that scores of frontal asymmetry have a narrower numerical range relative to other variables, readers should note that asymmetry scores presented in Table 1 have been truncated for presentation purposes but were not truncated during data analysis. Mean levels and ranges of frontal asymmetry scores for each episode showed that, consistent with our expectations about novelty and uncertainty, children showed greater asymmetry scores during the stranger conversation and experimenter conversation episodes relative to the stranger script episode. Although a range of positive (i.e., left asymmetry) and negative (i.e., right asymmetry) scores was observed, children on average displayed more left frontal asymmetry during the conversation episodes. A series of paired samples $t$-tests confirmed that asymmetry scores were greater for the stranger conversation relative the stranger script episode $(t=20.58, p<.01, d=0.16)$ and for the experimenter conversation relative to the stranger script episode $(t=2.97, p<.05, d=0.24)$. Frontal asymmetry scores did not differ between the two conversation episodes $(t=1.08, p>.10)$.

As indicated in Table 1, several significant sex differences emerged. Most notably, girls had more negative asymmetry scores (i.e., right asymmetry) and boys had more positive asymmetry scores (i.e., left asymmetry) during the baseline (girls: $M=-0.009, S D=0.04$; boys: $M=0.019, S D=0.03$ ) and stranger script episodes (girls: $M=-0.001, S D=0.08$; boys: $M=0.049, S D=0.05$ ). Given these differences, sex of participant was used as a covariate for analyses including the EEG variables.

Table 1

Means and standard deviations for variables.

\begin{tabular}{lccrrrr}
\hline & Minimum & Maximum & \multicolumn{1}{c}{$M$} & $S D$ & $t_{\text {twin }}$ & $t_{\text {sex }}$ \\
\hline Maternal negative affect $^{\mathrm{a}}$ & 2.79 & 46.00 & 22.74 & 9.93 & 0.76 & 0.80 \\
Overanxious behavior & 0.00 & 0.83 & 0.32 & 0.18 & -0.54 & -0.32 \\
Morning cortisol & -3.38 & 0.08 & -1.17 & 0.48 & 0.12 & -1.26 \\
Afternoon cortisol & -3.29 & -0.86 & -2.49 & 0.45 & -0.14 & 0.37 \\
Evening cortisol & -4.66 & -1.26 & -3.38 & 0.68 & -0.05 & $1.74^{\dagger}$ \\
Baseline frontal asymmetry & -0.12 & 0.09 & $0.01^{\mathrm{b}}$ & 0.04 & 0.55 & $3.35^{* *}$ \\
Stranger script frontal asymmetry & -0.18 & 0.24 & 0.02 & 0.07 & -0.39 & $3.22^{* *}$ \\
Stranger conversation frontal asymmetry & -0.18 & 0.14 & 0.01 & 0.06 & -0.51 & $1.82^{\dagger}$ \\
Experimenter conversation frontal asymmetry & -0.16 & 0.11 & 0.01 & 0.06 & -1.05 & 0.48 \\
Slopes of change in cortisol & -2.05 & 0.76 & -1.11 & 0.46 & -0.10 & $1.95^{\dagger}$ \\
Slopes of change in frontal asymmetry & -0.04 & 0.08 & 0.01 & 0.02 & 0.62 & $4.34^{* *}$ \\
\hline
\end{tabular}

Note. $N=98$. Variables are on separate scales and are not intended for comparisons across measures or collection methods. $t_{\text {twin }}$ is the result of an independent samples $t$-test conducted to test for twin differences in variables. $t_{\text {sex }}$ is the result of an independent samples $t$-test conducted to test for sex differences in variables.

a Imputation models do not force imputed scores into an interval scale, which occasionally results in a subset of values that are not whole numbers. We prefer to use the true imputed values rather than rounding to the nearest whole numbers.

b Values are truncated to two decimal places for presentation purposes. However, given the small scale of the EEG data, asymmetry values included 14 decimal places for analyses.

$\dagger p<.10$.

$p<.01$. 
Table 2

Bivariate correlations among the primary variables.

\begin{tabular}{|c|c|c|c|c|c|c|c|c|c|c|}
\hline & 1 & 2 & 3 & 4 & 5 & 6 & 7 & 8 & 9 & 10 \\
\hline \multicolumn{11}{|l|}{ 1. Maternal negative affect } \\
\hline 2. Overanxious behavior & .02 & & & & & & & & & \\
\hline 3. Morning cortisol & $-.45^{* *}$ & -.05 & & & & & & & & \\
\hline 4. Afternoon cortisol & -.17 & .12 & .16 & & & & & & & \\
\hline 5. Evening cortisol & .35 & $.28^{* *}$ & $-.24^{*}$ & $.42^{* *}$ & & & & & & \\
\hline 6. Baseline frontal asymmetry & $-.26^{*}$ & -.12 & -.05 & -.09 & -.10 & & & & & \\
\hline $\begin{array}{l}\text { 7. Stranger script frontal } \\
\text { asymmetry }\end{array}$ & $-.43^{* *}$ & -.05 & .09 & -.01 & .09 & $.63^{* *}$ & & & & \\
\hline $\begin{array}{l}\text { 8. Stranger conversation frontal } \\
\text { asymmetry }\end{array}$ & $-.31^{* *}$ & -.07 & .08 & .02 & .02 & $.63^{* *}$ & $.77^{* *}$ & & & \\
\hline $\begin{array}{l}\text { 9. Experimenter conversation } \\
\text { frontal asymmetry }\end{array}$ & $-.32^{* *}$ & -.15 & -.11 & -.02 & -.02 & $.59^{* * *}$ & $.72^{* *}$ & $.75^{* *}$ & & \\
\hline 10. Slopes of change in cortisol & $.49^{* *}$ & $.23^{*}$ & -.70 & $.23^{*}$ & $.86^{* *}$ & -.05 & .02 & -.03 & .04 & \\
\hline $\begin{array}{l}\text { 11. Slopes of change in frontal } \\
\text { asymmetry }\end{array}$ & $.29^{*}$ & -.13 & -.19 & -.09 & $-.24^{*}$ & $-.33^{* *}$ & $-.64^{* *}$ & $-.34^{* *}$ & .06 & .10 \\
\hline
\end{tabular}

Note. $N=98$.

${ }^{*} p<.05$.

** $p<.01$.

Table 3

Model fit statistics for mixed ANOVA models.

\begin{tabular}{|c|c|c|c|c|}
\hline \multirow[t]{2}{*}{ Model } & \multicolumn{2}{|c|}{ Diurnal cortisol } & \multicolumn{2}{|c|}{ Alpha asymmetry } \\
\hline & AIC & BIC & AIC & $\mathrm{BIC}$ \\
\hline Random intercepts only & 438.7 & 443.7 & -696.8 & -692.2 \\
\hline Random intercepts + random time & 411.3 & 421.3 & -699.8 & -690.6 \\
\hline Random intercepts + random time + random maternal negativity & 409.9 & 424.8 & -696.8 & -690.6 \\
\hline
\end{tabular}

Note. Italics indicate model determined to have best overall fit.

Nonsignificant differences between twins for all variables provided assurance that twin groupings, used to control for twins within families, were unrelated to measures of frontal asymmetry or diurnal cortisol.

Table 2 shows the bivariate correlations among the variables. Similar to past work, correlations between cortisol measures varied with time of day. In contrast, measures of frontal asymmetry were significantly correlated across all episodes. Measures of cortisol were unrelated to measures of frontal asymmetry. Maternal negativity was associated with greater right frontal asymmetry across all episodes but was inconsistently related to diurnal cortisol levels. Frontal asymmetry was not directly associated with overanxious behaviors, which is not uncommon for situations in which moderation (e.g., by maternal negativity) may be present (Aiken \& West, 1991).

\section{Childhood diurnal cortisol and maternal negative affect during infancy}

Cortisol samples were arranged in the order in which samples were collected, starting with morning samples and ending with evening samples. The final model for diurnal cortisol at age 7 years (fit statistics shown in Table 3) showed the expected decline in cortisol levels over time, $F(1,87)=663.58$, $p<.01$ (Table 4). However, this effect was subsumed in a significant interaction with maternal negative affect, $F(1,87)=29.08, p<.01$. We probed this interaction by reexamining the slope for diurnal cortisol at low $(-1 S D)$ and high $(+1 S D)$ levels of maternal negative affect (Aiken \& West, 1991). Recentering maternal negative affect allowed us to probe the interaction using a continuous variable and prevented the need to create arbitrary groups. Probing the interaction in this manner revealed greater decreases in age 7 cortisol across the day for children at low levels of maternal negative affect during infancy $(B=-1.330, S E(B)=0.061, \beta=-1.329, p<.01)$ relative to high levels of maternal negative during infancy $(B=-0.864, \operatorname{SE}(B)=0.061, \beta=-0.885, p<.01)$ (Fig. 1 ). 
Table 4

Diurnal cortisol and maternal negative affect.

\begin{tabular}{lllc}
\hline & $B$ & $S E(B)$ & $t$ \\
\hline Time (cortisol slope) & -1.097 & 0.043 & $-25.57^{* *}$ \\
Maternal negative affect & -0.025 & 0.004 & $-5.75^{* *}$ \\
Time * Maternal Negative Affect & 0.023 & 0.004 & $5.39^{* *}$ \\
\hline
\end{tabular}

Note. Because an $R^{2}$ calculation is not a part of the estimation procedure for mixed models, we calculated $R^{2}$ as the decrease in the proportion of error variance between the final model and an intercepts-only model. $\Delta R^{2}=.87$.

** $p<.01$.

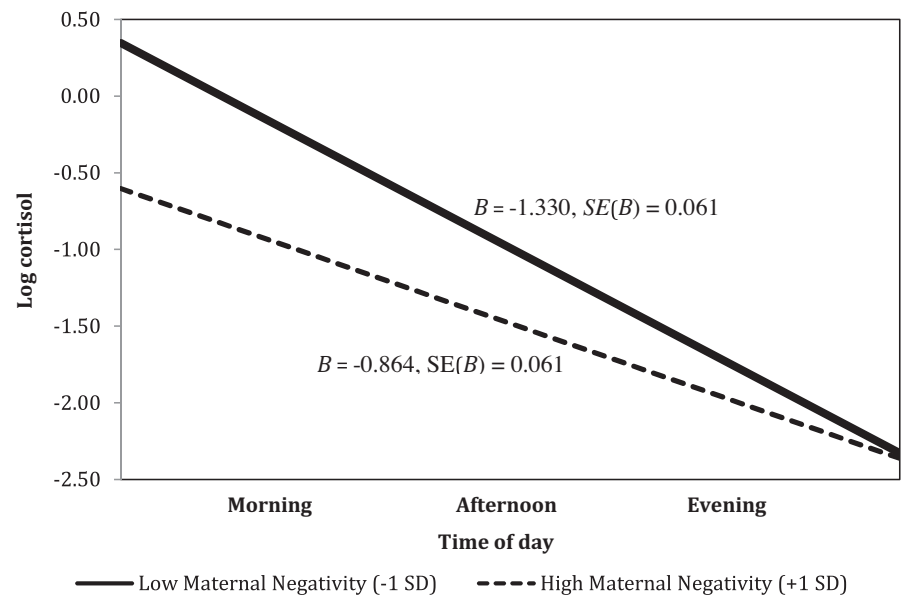

Fig. 1. Maternal negativity during infancy is linked to diurnal cortisol rhythm during childhood.

\section{Childhood frontal asymmetry across contexts and maternal negative affect during infancy}

The ordering for asymmetry scores was informed by the literature on asymmetry and coping. Although all contexts presented some degree of challenge in the form of novelty and/or uncertainty, we hypothesized that least left frontal asymmetry would be apparent in the conversation episode, which involved both high novelty (novel stranger or experimenter) and uncertainty (the expectations for children's role in the interaction), which may diminish active coping. In contrast, we expected that the greatest left frontal asymmetry would be apparent in the stranger script episode, which involved only moderate novelty (the stranger had been seen previously) and uncertainty (no requirements for children to participate in the episode). Notably, this theoretically based ordering of the episodes is consistent with the episode-based differences in frontal asymmetry reported above.

Similar to procedures followed in previous work (Buss, 2011; Calkins, Blandon, Williford, \& Keane, 2007), ordering the episodes in this way allows for a meaningful interpretation of the linear slope such that positive slopes indicated the expected increases in frontal asymmetry scores (i.e., more left frontal asymmetry) as contexts change. In contrast, flattened slopes indicated an absence of the expected increase in active approach-oriented coping across contexts. The final model included random intercepts and random slopes for both time and maternal negative affect (Table 3), again suggesting significant individual variation in both starting levels and slopes of change for infants.

As shown in Table 5, the expected increases in left frontal asymmetry across contexts were significant, $F(1,71)=11.03, p<.01$. Again, the effect of time was subsumed in a significant interaction with maternal negative affect during infancy, $F(1,71)=6.33, p=.01$. Probing the continuous slope of change in frontal asymmetry at low $(-1 S D)$ and high $(+1 S D)$ levels of maternal negative affect revealed that 
Table 5

Frontal asymmetry and maternal negative affect.

\begin{tabular}{|c|c|c|c|c|c|c|}
\hline & \multicolumn{3}{|c|}{ No control } & \multicolumn{3}{|c|}{ Control for baseline } \\
\hline & $B$ & $S E(B)$ & $t$ & $B$ & $S E(B)$ & $t$ \\
\hline Baseline asymmetry & - & - & - & 0.793 & 0.142 & $5.59^{* *}$ \\
\hline Sex & -0.017 & 0.011 & -1.47 & 0.001 & 0.010 & 0.12 \\
\hline Time (slope across contexts) & 0.009 & 0.003 & $3.32^{* *}$ & 0.009 & 0.003 & $3.32^{* *}$ \\
\hline Maternal negative affect & -0.001 & 0.001 & $-1.98^{*}$ & -0.001 & 0.001 & -1.13 \\
\hline Time * Maternal Negative Affect & -0.001 & 0.000 & $-2.52^{*}$ & -0.001 & 0.000 & $-2.52^{*}$ \\
\hline
\end{tabular}

Note. Because $R^{2}$ is not a part of the estimation procedure for mixed models, we calculated $\Delta R^{2}$ as the decrease in the proportion of error variance between the final model and an intercepts-only model. $\Delta R^{2}=.17$ for both models.

${ }^{*} p<.05$.

** $p<.01$.

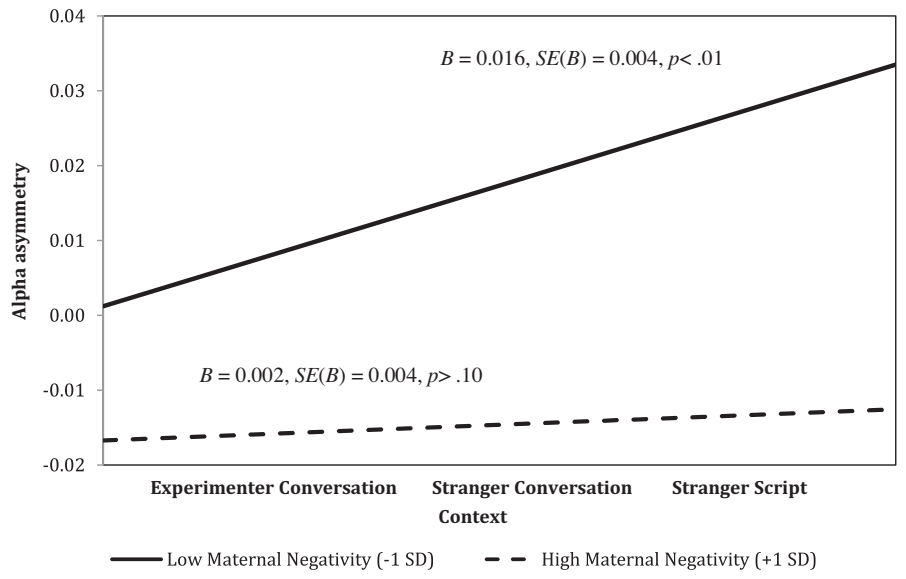

Fig. 2. Maternal negativity during infancy is linked to frontal asymmetry during childhood.

when levels of maternal negative affect during infancy were low, frontal asymmetry scores increased in the expected manner $(B=0.016, S E(B)=0.004, \beta=0.268, p<.01)$. However, no significant increases in frontal asymmetry scores across contexts were observed when levels of maternal negative affect during infancy were high $(B=0.002, S E(B)=0.004, \beta=0.045, p>.10)$ (Fig. 2). As shown in Table 5, effects remained significant when baseline levels of EEG were controlled.

\section{Psychophysiological dysregulation and anxious behaviors}

In our final analysis, we examined whether the regulation as assessed by slopes of diurnal cortisol and frontal asymmetry were related to parent-reported anxious behaviors in children. Overanxious behaviors were positively related to cortisol slope $(r=.23, p<.05)$, suggesting that smaller declines in cortisol across the day were related to more anxious behaviors. In contrast, overanxious behaviors were unrelated to slopes of frontal asymmetry $(r=.13, p>.10)$.

\section{Post hoc analyses}

Given the significant correlation between cortisol slopes and anxious behaviors, we conducted a post hoc test of the possibility that dysregulated cortisol slopes serve as mechanisms of risk for anxious behaviors. To do this, we tested slopes of change in diurnal cortisol as mediators of an association between maternal negative affect during infancy and overanxious behaviors during middle childhood 


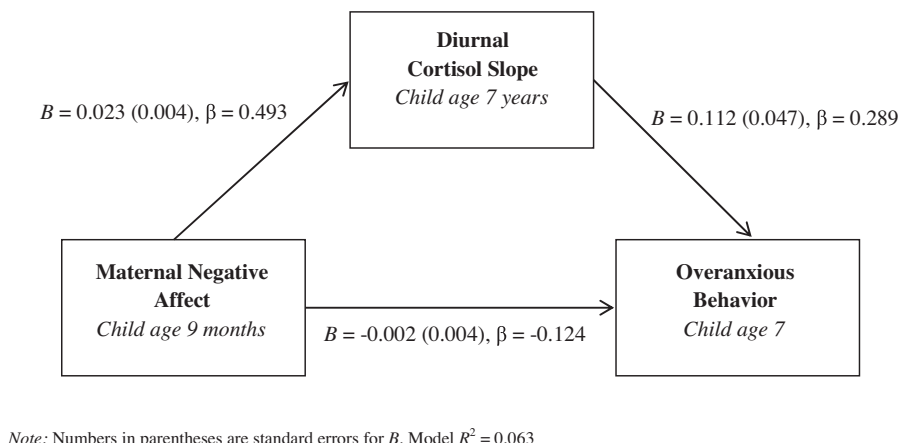

Fig. 3. Diurnal cortisol slopes mediate an association between maternal negative affect during infancy and overanxious behaviors during childhood. Numbers in parentheses are standard errors for $B$. Model $R^{2}=.063$.

(Fig. 3). The significance of the indirect effect was evaluated using an ordinary least squares regression model with an automated bootstrapping ( $n$ bootstraps $=1000$ ) procedure (PROCESS 2.01 beta). Indeed, we observed a modest $\left(\kappa^{2}=.13\right.$; Preacher \& Kelley, 2011) indirect effect of maternal negative affect on overanxious symptoms; the 95\% confidence intervals for this effect did not contain zero $(\beta=0.14$, $S E=0.06, C I_{\text {lower }}=0.03, C I_{\text {upper }}=0.28$ ), suggesting the presence of statistically significant mediation.

Given that diurnal cortisol slope and overanxious behaviors were measured at the same age, we also tested whether overanxious behaviors during middle childhood mediated a relation between maternal negative affect during infancy and diurnal cortisol slopes. However, results showed that overanxious behaviors did not mediate this association $\left(\beta=0.00, S E=0.03, C I_{\text {lower }}=-0.05\right.$, $C I_{\text {upper }}=0.07$ ), indicating that the previous model including diurnal cortisol slopes as a mediator fit our data best.

\section{Discussion}

High levels of maternal negative affect during infancy predicted disruptions in psychophysiological systems that support active coping during middle childhood. We observed flattened slopes of diurnal cortisol and frontal asymmetry for 7-year-old children whose mothers reported greater negative affect during infancy. Findings are consistent with early sensitization theory given that maternal negative affect measured at 9 months of age predicted psychophysiological function in children more than 6 years later.

As hypothesized, when maternal negative affect during infancy was high, diurnal cortisol slopes were flat. Although maternal negativity is unlikely to directly influence diurnal cortisol rhythms, negative affect in mothers may affect HPA function via early parenting behaviors. During the first year of life, sensitive responsive behaviors from caregivers mitigate glucocorticoid responsivity (Gunnar, Larson, Hertsgaard, Harris, \& Brodersen, 1992; Spangler \& Schieche, 1998). In contrast, high levels of negative affect in mothers are associated with more negative parenting behaviors, including harshness, criticism, and rejection (Lovejoy et al., 2000). Harsh parenting practices, in turn, have been linked to alterations in HPA function and risk for negative psychosocial outcomes (Bugental, Martorell, \& Barraza, 2003). Thus, highly negative mothers may both employ more behaviors that lead to dysregulated physiological systems and fail to employ those maternal behaviors that can buffer against physiological dysregulation. In fact, in the larger sample of families participating at 9 months of age, ${ }^{5}$ scores of maternal negative affect were negatively correlated with two separate observations $\left(r_{669}=-.28\right.$ and

\footnotetext{
${ }^{5}$ Observational data were available for a subset of families who participated in the infant assessment. Although observational data would enhance the analyses presented here, the subset of families with parent-child interaction data during infancy had very little overlap with the families who participated in the 7-year assessment, resulting in a large amount of missing data. Thus, parent-reported negative affect data were used.
} 
$r_{243}=-.22, p s<.01$ ) of maternal sensitivity (NCAST-PCI [Parent-Child Interaction]; Sumner \& Spitz, 1994), supporting the idea that greater negativity in mothers is linked with fewer sensitive parenting behaviors. Maternal negativity was unrelated to factors of general family context such as socioeconomic status (Hollingshead index; $r_{335}=-.08, p>.10$ ).

To our knowledge, this is the first study to indicate that high levels of maternal negative affect during infancy are associated with slopes of frontal asymmetry during middle childhood. When levels of maternal negativity during infancy were high, children showed right frontal asymmetry across all contexts, suggesting that avoidance tendencies were unaltered by changes in contextual approach incentives. Consistent with theories of coping, children who experienced high levels of maternal negative affect may employ less approach-oriented coping, even when called for by the context, relative to other children. In contrast, when levels of maternal negativity during infancy were low, children showed greater left frontal asymmetry (higher asymmetry scores) as they engaged in more approach-oriented coping and less avoidance-oriented coping.

Given previous work associating left frontal asymmetry with approach-oriented behaviors (Davidson \& Irwin, 1999; Harmon-Jones \& Allen, 1998), the pattern of increasing asymmetry scores under conditions of low maternal negativity may reflect global tendencies for children of less negative parents to adopt approach-oriented coping strategies as contexts change. Our data suggest that these differences are most pronounced in contexts where the necessity of active interactions is most ambiguous. Under this interpretation, all children would be expected to similarly engage neural resources in unambiguous novel contexts. However, as contexts become more ambiguous, level of maternal negativity during infancy would predict the degree to which children adopt approachoriented coping behaviors such that lower levels of negativity are associated with greater propensities for approach.

This approach-related interpretation is consistent with our expectations for individual differences in the use of active coping strategies. Similar patterns of findings for the association of maternal negativity with active coping indicated by both HPA function and frontal asymmetry may reflect an overlap in the acquisition of self-regulatory skills linked to the development of the prefrontal cortex. Neural systems that support coping behaviors are relatively immature early in life; this immaturity leaves infants reliant on external coping resources (Gogtay et al., 2004; Huttenlocher, 1990). In cases where external support may be less available, such as in early environments characterized by high negative affect (Hoffman, Crnic, \& Baker, 2006), an inability to cope effectively may lay the foundation for a dysregulated template for stress responses. Mothers high in negative affect are also less effective at scaffolding coping strategies for young children (Hoffman et al., 2006). Thus, even as neural circuitry matures, children of mothers who are highly negative might not acquire independent coping strategies to the same extent as children of less negative mothers. Rather, it is possible that children of highly negative mothers may continue to rely on less sophisticated and less effective strategies from earlier in development (Mangelsdorf, Shapiro, \& Marzolf, 1995).

Although patterns of findings were similar for systems of neural and neuroendocrine regulation, slopes of change indicating regulation in each system were uncorrelated. Thus, children do not appear to be showing evidence of simultaneous disruptions in multiple systems that support active coping. One explanation for this is the vast individual differences that are present in stress responsivity in humans (McEwen, 1998), including broad variability in the perception of, evaluation of, and capacity for coping in challenging contexts. Another possible explanation stems from the idea that although multiple physiological systems are susceptible to the influences of the early environment, systems differ in the timing and degree of susceptibility (McEwen, 1998; Sapolsky, 1998). Thus, the point at which different biological systems become exhausted or disrupted, resulting in dysregulation, is unlikely to be the equivalent. From a biological perspective, differences in the timing and onset of dysregulation across coping systems would be protective, allowing organisms to continue to survive in a context where threats will continue to be encountered.

Our work does not account for levels of negative affect in mothers at the 7-year psychophysiological assessment. Thus, unmeasured concurrent negative affect might also contribute to patterns of psychophysiological dysregulation during middle childhood. Past work suggests some stability in levels of maternal negative affect between ages 2 and 5 years (Feng, Shaw, Skuban, \& Lane, 2007). Even so, it is perhaps more remarkable that maternal negativity during infancy was associated with 
psychophysiological substrates of coping in children more than 6 years later. Statistically, one would expect that such an association would diminish over time as additional sources of variability are introduced. Between 9 months and 7 years of age, these other sources of variability would include major events such as children becoming independently mobile and vocal, starting school, making friends outside of the home, developing relationships with teachers, and orienting to new environments, each of which may contribute to their capacity for effective coping. Thus, although some association between concurrent measures would be anticipated, the persistent role of an infant measure of maternal negative affect in predicting physiological systems during childhood is rare and certainly noteworthy. Of additional importance, these findings are consistent with theoretical depictions of infancy as a critical period for the sensitization of systems that support active coping for the overall acquisition of regulatory and coping skills (Gunnar, 2003; Kopp, 1982; Shirtcliff \& Ruttle, 2010).

An alternative explanation for the association between early maternal negative affect and children's patterns of dysregulation in biological systems suggests similar biological underpinnings for negative affect in mothers and biological dysregulation in children. Because children in our study were raised by their biological parents, biological and environmental contributions to children's development cannot be disentangled. Indeed, cortisol levels in children are heritable, with higher heritability estimates for morning than for evening levels (Bartels, de Geus, Kirschbaum, Sluyter, \& Boomsma, 2003; Van Hulle, Shirtcliff, Lemery-Chalfant, \& Goldsmith, 2012). EEG asymmetry is also moderately heritable (Anokhin, Heath, \& Myers, 2006; Coan, 2003). Thus, similar fluctuations in cortisol levels or frontal asymmetry may be influenced, to some extent, by shared genetic factors in mothers and children.

Nonetheless, substantial evidence also indicates that environmental factors modulate diurnal cortisol rhythms. For example, across the course of an intervention aimed at improving caregiving environments, diurnal cortisol activity in foster children between 3 and 6 years of age (i.e., children being reared by genetically unrelated adults) became more similar to that of typically developing children (Fisher, Stoolmiller, Gunnar, \& Burraston, 2007). The role of contextual factors in neural profiles has also been discussed (Davidson, Jackson, \& Kalin, 2000), although existing evidence suggests that shared genetic factors do not fully account for similarities in emotional profiles in mothers and infants (Field, Vega-Lahr, Goldstein, \& Scafidi, 1987). Thus, evidence for heritability does not negate the importance of the early environment for developing biological systems.

Finally, this work provided evidence that greater HPA dysregulation (i.e., flatter slopes of change) was associated with more overanxious behaviors in children. Moreover, a mediation analysis supported HPA dysregulation as a mechanism for the association of maternal negative affect during infancy with the development of anxiety problems. Indirect effects linking maternal negative affect with children's overanxious symptoms via disruptions in HPA regulation were modest. Again, this may reflect the more than 6-year lag between our maternal negativity measure and childhood outcomes. In this way, the longitudinal nature of our data provides a powerful test of HPA regulation as a mechanism in the development of overanxious behaviors in children (Collins \& Graham, 2002). Although clinical diagnoses are not available, this work shows that greater maternal negative affect during infancy predicts flatter slopes of diurnal cortisol and ultimately more overanxious behaviors at age 7 years. Concurrent measurement of psychophysiology and overanxious behaviors at age 7 precludes conclusions about causality; however, these links underscore the assertion that greater maternal negativity may disrupt active coping at the neuroendocrine level.

Unexpectedly, slopes of EEG asymmetry were uncorrelated with overanxious behaviors. Although baseline frontal asymmetry has shown robust associations with levels of anxiety, this finding has been less consistent for state-level asymmetry measures, which may be less sensitive to individual differences than are trait-level assessments (Coan et al., 2006). In addition, the existing literature suggests that cortisol is related to general efforts at adaptive coping (Gunnar, 1992); in contrast, we hypothesized that links between active coping and frontal asymmetry would occur specifically through approach orientation. Thus, it is possible that although frontal asymmetry may be disrupted by early maternal negativity, the manifestation of overanxious behaviors may also depend on a broad behavioral repertoire that includes both approach tendencies and a variety of other active coping behaviors (Carver et al., 1989). Additional work that includes assessments of a variety of coping behaviors will help to clarify this issue. 
Our study is not without limitations. First, our sample was relatively homogeneous. Although there is no evidence that the processes outlined here would be altered across race or ethnicity, we cannot directly test this possibility. Second, although we do not view the twin design as a limitation, we recognize that concerns may exist that twin samples do not represent the general population on one or more aspects of behavior, which may bias results in some systematic way. In fact, twins and non-twins frequently differ in factors such as gestational age at birth, birth weight, and rate of language acquisition early in life (Plomin, DeFries, McClearn, \& McGuffin, 2008). However, many of these differences decline and disappear by the early school years. Results from twin studies largely generalize to non-twins (Andrew et al., 2001); this generalization has been demonstrated for measures of biologically based individual differences (Goldsmith \& Campos, 1990) and psychopathology (Christensen, Vaupel, Holm, \& Yashin, 1995).

We have presented novel evidence that high levels of maternal negative affect disrupt physiological systems that may support active coping at the levels of neural (frontal asymmetry) and neuroendocrine (diurnal cortisol) function. Importantly, these effects were longitudinal such that measures of maternal negativity obtained when children were 9 months of age modulated children's psychophysiological regulation at 7 years of age. We showed that flattened slopes of change of diurnal cortisol were associated with concurrent measures of children's anxious behaviors. A mediation analysis supported the notion that dysregulated changes in diurnal cortisol may be one mechanism of heightened risk for anxiety problems.

\section{Acknowledgments}

Data collection for this project was supported by MH50560 (principal investigator [PI]: Goldsmith) from the National Institute of Mental Health. Infrastructure support was provided by P30 HD03352 and P50 MH084051. The writing of this article was supported by K01 MH100240 (PI: Brooker) from the National Institute of Mental Health. The content is solely the responsibility of the authors and does not necessarily represent the official views of the National Institutes of Health. We thank the families who participated in this study and the staff members who helped with the recruitment of study participants and data collection.

\section{References}

Aiken, L. S., \& West, S. G. (1991). Multiple regression: Testing and interpreting interactions. Thousand Oaks, CA: Sage.

Andrew, T., Hart, D. J., Snieder, H., de Lange, M., Spector, T. D., \& MacGregor, A. J. (2001). Are twins and singletons comparable? A study of disease-related and lifestyle characteristics in adult women. Twin Research and Human Genetics, 4, 464-477.

Anokhin, A. P., Heath, A. C., \& Myers, E. (2006). Genetic and environmental influences on frontal EEG asymmetry: A twin study. Biological Psychology, 71, 289-295.

Bartels, M., de Geus, E. C., Kirschbaum, C., Sluyter, F., \& Boomsma, D. (2003). Heritability of daytime cortisol levels in children. Behavior Genetics, 33, 421-433.

Baving, L., Laucht, M., \& Schmidt, M. H. (1999). Atypical frontal brain activation in ADHD: Preschool and elementary school boys and girls. Journal of the American Academy of Child and Adolescent Psychiatry, 38, 1363-1371.

Biederman, J., Hirshfeld-Becker, D. R., Rosenbaum, J. F., Herot, C., Friedman, D., Snidman, N., ... Faraone, S. V. (2001). Further evidence of association between behavioral inhibition and social anxiety in children. American Journal of Psychiatry, 158, 1673-1679.

Blalock, J. A., \& Joiner, T. E. Jr., (2000). Interaction of cognitive avoidance coping and stress in predicting depression/anxiety. Cognitive Therapy and Research, 24, 47-65.

Bryden, M. P. (1982). Laterality: Functional asymmetry in the intact brain. New York: Academic Press.

Bugental, D. B., Martorell, G. A., \& Barraza, V. (2003). The hormonal costs of subtle forms of infant maltreatment. Hormones and Behavior, 43, 237-244.

Buss, K. A. (2011). Which fearful toddlers should we worry about? Context, fear regulation, and anxiety risk. Developmental Psychology, 47, 804-819.

Buss, K. A., Schumacher, J. R. M., Dolski, I., Kalin, N. H., Goldsmith, H. H., \& Davidson, R. J. (2003). Right frontal brain activity, cortisol, and withdrawal behavior in 6-month-old infants. Behavioral Neuroscience, 117, 11-20.

Calkins, S. D., Blandon, A. Y., Williford, A. P., \& Keane, S. P. (2007). Biological, behavioral, and relational levels of resilience in the context of risk for early childhood behavior problems. Development and Psychopathology, 19, 675-700.

Carlson, M., \& Earls, F. (1997). Psychological and neuroendocrinological sequelae of early social deprivation in institutionalized children in Romania. Annals of the New York Academy of Sciences, 807, 419-428.

Carver, C. S., Scheier, M. F., \& Weintraub, J. K. (1989). Assessing coping strategies: A theoretically based approach. Journal of Personality and Social Psychology, 56, 267-283. 
Christensen, K., Vaupel, J. W., Holm, N. V., \& Yashin, A. I. (1995). Mortality among twins after age 6: Fetal origins hypothesis versus twin method. British Medical Journal, 310, 432-436.

Chronis-Tuscano, A., Degnan, K. A., Pine, D. S., Perez-Edgar, K., Henderson, H. A., Diaz, Y., ... Fox, N. A. (2009). Stable early maternal report of behavioral inhibition predicts lifetime social anxiety disorder in adolescence. Journal of the American Academy of Child and Adolescent Psychiatry, 48, 928-935.

Coan, J. A. Jr., (2003). The heritability of trait frontal EEG asymmetry and negative emotionality: Sex differences and genetic nonadditivity (unpublished doctoral dissertation). University of Arizona.

Coan, J. A., Allen, J. J. B., \& McKnight, P. E. (2006). A capability model of individual differences in frontal EEG asymmetry. Biological Psychology, 72, 198-207.

Collins, L. M., \& Graham, J. W. (2002). The effect of the timing and spacing of observations in longitudinal studies of tobacco and other drug use: Temporal design considerations. Drug and Alcohol Dependence, 68(Suppl. 1), S85-S96.

Davidson, R. J. (2002). Anxiety and affective style: Role of prefrontal cortex and amygdala. Biological Psychiatry, 51, 68-80.

Davidson, R. J., \& Irwin, W. (1999). The functional neuroanatomy of emotion and affective style. Trends in Cognitive Sciences, 3 , 11-21.

Davidson, R. J., Jackson, D. C., \& Kalin, N. H. (2000). Emotion, plasticity, context, and regulation: Perspectives from affective neuroscience. Psychological Bulletin, 126, 890-909.

Dawson, G., Frey, K., Panagiotides, H., Yamada, E., Hessl, D., \& Osterling, J. (1999). Infants of depressed mothers exhibit atypical frontal electrical brain activity during interactions with mother and with a familiar, nondepressed adult. Child Development, 70, 1058-1066.

Dawson, G., Frey, K., Self, J., Panagiotides, H., Hessl, D., Yamada, E., et al (1999). Frontal brain electrical activity in infants of depressed and nondepressed mothers: Relation to variations in infant behavior. Development and Psychopathology, 11, 589-605.

Degnan, K. A., Almas, A. N., \& Fox, N. A. (2010). Temperament and the environment in the etiology of childhood anxiety. Journal of Child Psychology and Psychiatry, 51, 497-517.

Eisenberg, N., Gershoff, E. T., Fabes, R. A., Shepard, S. A., Cumberland, A. J., Losoya, S. H., ... Murphy, B. C. (2001). Mother's emotional expressivity and children's behavior problems and social competence: Mediation through children's regulation. Developmental Psychology, 37, 475-490.

Enders, C. (2010). Applied missing data analysis. New York: Guilford.

Essex, M., Boyce, W. T., Goldstein, L. H., Armstrong, J. M., Kraemer, H., Kupfer, D. J., et al (2002). The confluence of mental, physical, social, and academic difficulties in middle childhood: II. Developing the MacArthur Health and Behavior Questionnaire. Journal of the American Academy of Child and Adolescent Psychiatry, 41, 588-603.

Essex, M. J., Klein, M. H., Cho, E., \& Kalin, N. H. (2002). Maternal stress beginning in infancy may sensitize children to later stress exposure: Effects on cortisol and behavior. Biological Psychiatry, 52, 776-784.

Essex, M. J., Klein, M. H., Cho, E., \& Kraemer, H. C. (2003). Exposure to maternal depression and marital conflict: Gender differences in children's later mental health symptoms. Journal of the American Academy of Child and Adolescent Psychiatry, 42, 728-737.

Feng, X., Shaw, D. S., Skuban, E. M., \& Lane, T. (2007). Emotional exchange in mother-child dyads: Stability, mutual influence, and associations with maternal depression and child problem behavior. Journal of Family Psychology, 21, 714-725.

Field, T., Vega-Lahr, N., Goldstein, S., \& Scafidi, F. (1987). Face-to-face interaction behavior across early infancy. Infant Behavior and Development, 10, 111-116.

Fisher, P. A., Stoolmiller, M., Gunnar, M. R., \& Burraston, B. O. (2007). Effects of a therapeutic intervention for foster preschoolers on diurnal cortisol activity. Psychoneuroendocrinology, 32, 892-905.

Fox, N. A., Rubin, K. H., Calkins, S. D., Marshall, T. R., Coplan, R. J., Porges, S. W., et al (1995). Frontal activation asymmetry and social competence at four years of age. Child Development, 66, 1770-1784.

Gogtay, N., Giedd, J. N., Lusk, L., Hayashi, K. M., Greenstein, D., Vaituzis, A. C., ... Thompson, P. M. (2004). Dynamic mapping of human cortical development during childhood through early adulthood. Proceedings of the National Academy of Sciences of the United States of America, 101, 8174-8179.

Goldsmith, H. H., \& Campos, J. J. (1990). The structure of temperamental fear and pleasure in infants: A psychometric perspective. Child Development, 61, 1944-1964.

Graham, J. W. (2009). Missing data analysis: Making it work in the real world. Annual Review of Psychology, 60, 549-576.

Graham, J. W., \& Schafer, J. L. (1999). On the performance of multiple imputation for multivariate data with small sample size. In R. Hoyle (Ed.), Statistical strategies for small sample research (pp. 1-29). Thousand Oaks, CA: Sage.

Greaves-Lord, K., Ferdinand, R. F., Oldehinkel, A. J., Sondeijker, F. E. P. L., Ormel, J., \& Verhulst, F. C. (2007). Higher cortisol awakening response in young adolescents with persistent anxiety problems. Acta Psychiatrica Scandinavica, 116, $137-144$.

Gunnar, M. R. (1992). Reactivity of the hypothalamic-pituitary-adrenocortical system to stressors in normal infants and children. Pediatrics, 90, 491-497.

Gunnar, M. R. (2003). Integrating neuroscience and psychological approaches in the study of early experiences. Annals of the New York Academy of Sciences, 1008, 238-247.

Gunnar, M. R., Larson, M. C., Hertsgaard, L., Harris, M. L., \& Brodersen, L. (1992). The stressfulness of separation among ninemonth-old infants: Effects of social context variables and infant temperament. Child Development, 63, 290-303.

Gunnar, M., \& Quevedo, K. (2007). The neurobiology of stress and development. Annual Review of Psychology, 58, 145-173.

Gunnar, M. R., \& Vazquez, D. M. (2001). Low cortisol and a flattening of expected daytime rhythm: Potential indices of risk in human development. Development and Psychopathology, 13, 515-538.

Hale, T. S., Smalley, S. L., Dang, J., Hanada, G., Macion, J., McCracken, J. T., ... Loo, S. K. (2010). ADHD familial loading and abnormal EEG alpha asymmetry in children with ADHD. Journal of Psychiatric Research, 44, 605-615.

Harmon-Jones, E., \& Allen, J. J. B. (1998). Anger and frontal brain activity: EEG asymmetry consistent with approach motivation despite negative affective valence. Journal of Personality and Social Psychology, 74, 1310-1316.

Heim, C., Ehlert, U., \& Hellhammer, D. H. (2000). The potential role of hypocortisolism in the pathophysiology of stress-related bodily disorders. Psychoneuroendocrinology, 25, 1-35. 
Hoffman, C., Crnic, K. A., \& Baker, J. K. (2006). Maternal depression and parenting: Implications for children's emergent emotion regulation and behavioral functioning. Parenting, 6, 271-295.

Huttenlocher, P. R. (1990). Morphometric study of human cerebral cortex development. Neuropsychologia, 28, $517-527$.

Jones, N. A., Field, T., \& Davalos, M. (2000). Right frontal EEG asymmetry and lack of empathy in preschool children of depressed mothers. Child Psychiatry and Human Development, 30, 189-204.

Kagan, J. (1994). Galen's prophecy: Temperament in human nature. New York: Basic Books.

Kopp, C. B. (1982). Antecedents of self-regulation: A developmental perspective. Developmental Psychology, 18, 199-214.

Laborit, H. (1976). On the mechanism of activation of the hypothalamo-pituitary-adrenal reaction to changes in the environment (the "alarm reaction"). Resuscitation, 5(1), 19-30.

Larson, M. C., White, B. P., Cochran, A., Donzella, B., \& Gunnar, M. (1998). Dampening of the cortisol response to handling at 3 months in human infants and its relation to sleep, circadian cortisol activity, and behavioral distress. Developmental Psychobiology, 33, 327-337.

Lazarus, R. S. (1966). Psychological stress and the coping process. New York: McGraw-Hill.

Locke, R. L., Davidson, R. J., Kalin, N. H., \& Goldsmith, H. H. (2009). Children's context inappropriate anger and salivary cortisol. Developmental Psychology, 45, 1284-1297.

Lovejoy, M. C., Graczyk, P. A., O’Hare, E., \& Neuman, G. (2000). Maternal depression and parenting behavior: A meta-analytic review. Clinical Psychology Review, 20, 561-592.

Mangelsdorf, S. C., Shapiro, J. R., \& Marzolf, D. (1995). Developmental and temperamental differences in emotion regulation in infancy. Child Development, 66, 1817-1828.

McEwen, B. S. (1998). Stress, adaptation, and disease. Annals of the New York Academy of Sciences, 840, 33-44.

McManis, M. H., Kagan, J., Snidman, N. C., \& Woodward, S. A. (2002). EEG asymmetry, power, and temperament in children. Developmental Psychobiology, 41, 169-177.

Miller, D. B., \& O’Callaghan, J. P. (2002). Neuroendocrine aspects of the response to stress. Metabolism, 51, 5-10.

Munck, A., Guyre, P. M., \& Holbrook, N. J. (1984). Physiological functions of glucocorticoids in stress and their relation to pharmacological actions. Endocrine Reviews, 5, 25-44.

Plomin, R., DeFries, J. C., McClearn, G. E., \& McGuffin, P. (2008). Behavioral genetics (5th ed.). New York: Worth.

Preacher, K. J., \& Kelley, K. (2011). Effect size measures for mediation models: Quantitative strategies for communicating indirect effects. Psychological Methods, 16, 93-115.

Sapolsky, R. (1998). Why zebras don't get ulcers (3rd ed.). New York: Henry Holt.

Schmidt, N. L., Van Hulle, C. A., Brooker, R. J., Meyer, L. R., Lemery-Chalfant, K., \& Goldsmith, H. H. (2013). Wisconsin twin research: Early development, childhood psychopathology, autism, and sensory over-responsivity. Twin Research and Human Genetics, 16, 376-384.

Shirtcliff, E. A., \& Essex, M. J. (2008). Concurrent and longitudinal associations of basal and diurnal cortisol with mental health symptoms in early adolescence. Developmental Psychobiology, 50, 690-703.

Shirtcliff, E. A., \& Ruttle, P. (2010). Immunological and neuroendocrine dysregulation following early deprivation and stress. In K. Heinz Brisch (Ed.), Attachment and early disorders of development. Klett-Cotta: Munich, Germany.

Spangler, G., \& Schieche, M. (1998). Emotional and adrenocortical responses of infants to the Strange Situation: The differential function of emotional expression. International Journal of Behavioral Development, 22, 681-706.

Sumner, G., \& Spitz, A. (1994). Caregiver/parent-child interaction teaching manual. Seattle, WA: NCAST Publications.

Thompson, R. A. (1994). Emotion regulation: A theme in search of definition. Monographs of the Society for Research in Child Development, 59 (2-3, Serial No. 240).

Tops, M., van der Pompe, G., Wijers, A. A., Den Boer, J. A., Meijman, T. F., \& Korf, J. (2004). Free recall of pleasant words from recency positions is especially sensitive to acute administration of cortisol. Psychoneuroendocrinology, 29, 327-338.

Van den Bergh, B. R. H., Van Calster, B., Pinna Puissant, S., \& Van Huffel, S. (2008). Self-reported symptoms of depressed mood, trait anxiety, and aggressive behavior in post-pubertal adolescents: Associations with diurnal cortisol profiles. Hormones and Behavior, 54, 253-257.

Van Hulle, C. A., Shirtcliff, E. A., Lemery-Chalfant, K., \& Goldsmith, H. H. (2012). Genetic and environmental influences on individual differences in cortisol level and circadian rhythm in middle childhood. Hormones and Behavior, 62, 36-42.

Watson, D., Clark, L. A., \& Tellegen, A. (1988). Development and validation of brief measures of positive and negative affect: The PANAS scales. Journal of Personality and Social Psychology, 54, 1063-1070.

Weinberg, M. K., \& Tronick, E. Z. (1998). Emotional characteristics of infants associated with maternal depression and anxiety. Pediatrics, 102(Suppl. E1), 1298-1304. 